\title{
Clinical Characteristics, Management, and Natural History of Chronic Inducible Urticaria in a Pediatric Cohort
}

\author{
Laura May Miles $^{a} \quad$ Sofianne Gabrielli ${ }^{a} \quad$ Michelle Le ${ }^{a} \quad$ Elena Netchiporouk ${ }^{b}$ \\ Sharon Baum $^{c}$ Shoshana Greenberger ${ }^{d}$ Luis F. Ensina ${ }^{e}$ Fatemeh Jafarian $^{a}$ \\ Xun Zhang $^{f}$ Moshe Ben-Shoshan ${ }^{a}$ \\ ${ }^{a}$ Division of Pediatric Allergy and Clinical Immunology, Department of Pediatrics, McGill University Health \\ Center, Montreal, QC, Canada; ${ }^{b}$ Division of Dermatology, McGill University, Montreal, QC, Canada; \\ 'Department of Dermatology, Chaim Sheba Medical Center, Sackler School of Medicine, Tel-Aviv University, \\ Tel Hashomer, Israel; ' Department of Dermatology, Sheba Medical Center, Ramat-Gan, Israel; ${ }^{\text {eDepartment of }}$ \\ Pediatrics, Federal University of Sao Paulo, Sao Paulo, Brazil; ${ }^{f}$ Centre for Outcome Research and Evaluation, \\ Research Institute of McGill University Health Centre, Montreal, QC, Canada
}

\section{Keywords}

Chronic urticaria $\cdot$ Chronic inducible urticaria $\cdot$ Triggers

\begin{abstract}
Background: Some forms of chronic urticaria (CU) can be specifically attributed to a response to a definite trigger, referred to as chronic inducible urticaria (CIndU). We aimed to assess the demographics, clinical characteristics, comorbidities, natural history, and management of pediatric patients with CIndU. Methods: Over a 6-year period, children presenting to the allergy clinic at the Montreal Children's Hospital $(\mathrm{MCH})$ with CIndU were prospectively recruited. CU was defined as the presence of wheals and/or angioedema, occurring for at least 6 weeks. A standardized diagnostic test was used to establish the presence of a specific form of urticaria. Resolution was defined as the absence of hives for 1 year without treatment. Results: Sixty-four patients present-
\end{abstract}

ed with CIndU, of which $51.6 \%$ were male, with a median age of 12.5 (interquartile range $7.3,15.9$ ) years. Cold CU and cholinergic CU were the most common subtypes (60.3 and $41.3 \%$, respectively). Basophil counts were undetectable in $48.4 \%$ of the cases, and C-reactive protein levels were elevated in $7.8 \%$ of patients. Of all cases, $71.4 \%$ were controlled with second-generation antihistamines. The resolution rate was of $45.3 \%$ (95\% confidence interval 33.1-57.5\%), based on per-protocol population within the 6-year course of the study. Resolution was more likely in patients who presented with well-controlled urticaria control test scores and elevated CD63 counts and in those suffering from thyroid comorbidity. Conclusion: The natural history of CIndU resolution in pediatric patients was relatively low and was associated with elevated CD63 levels, as well as thyroid comorbidity.
Correspondence to:

Laura May Miles, Laura.miles@mail.mcgill.ca 


\section{Introduction}

Chronic urticaria (CU) is characterized as hives and/ or angioedema occurring for a period of at least 6 weeks [1]. Previous studies have demonstrated that between 0.1 and $1.0 \%$ of children present with CU [2,3]. A recent study conducted in Korea reported that this prevalence may increase [4]. In up to $80 \%$ of cases, CU occurs without exposure to a definite trigger and is defined as chronic spontaneous urticaria (CSU) [5]. A minority of CU (20\%) can be specifically attributed to a response to a definite trigger and is commonly referred to as chronic inducible urticaria (CIndU), whereas approximately $10 \%$ of patients present with both types of CU $[6,7]$. These percentages are similar in pediatric cohorts [8]. Subtypes of CIndU include physical urticaria in response to a physical stimulus (e.g., dermatographic, cold, solar, pressure, heat, or vibration), or cholinergic urticaria [9].

There are little data on management, demographics, diagnosis, triggers, and resolution of CIndU in children. We aimed to assess the demographics, clinical characteristics, comorbidities, family history, natural history, and management of pediatric patients with CIndU who presented to the Montreal Children's Hospital (MCH).

\section{Materials and Method}

Over a 6-year period, between 2013 and 2019, children (0-18 years) presenting to the allergy clinic at the MCH, a GA ${ }^{2} \mathrm{LEN} \mathrm{Ur}$ ticaria Center of Reference and Excellence (UCARE), with CIndU were prospectively recruited as part of our urticaria registry. The urticaria registry is a cohort study recruiting children with $\mathrm{CU}$ [10]. Approval was received by the McGill University Health Centre Research Ethics Board, from the Centre for Applied Ethics (REB 12-255 GEN, June 3, 2013). Written informed consent was obtained from parents or legal guardians of patients, as well as patients older than 7 years. CU was defined as the presence of wheals and/or angioedema, occurring for at least 6 weeks. Diagnosis of CIndU was based on clinical history and a standardized provocation test, as previously described [11]. The trigger was used to establish the presence of a specific form of CIndU when an inducible form was suspected according to patients' history [11]. For example, for cholinergic urticaria, patients were required to perform physical exercise for $30 \mathrm{~min}$. For cold urticaria, the ice cube test was performed in which we exposed the patients to an ice cube in a thin plastic bag for $5 \mathrm{~min}$ [9]. A standardized data entry form documenting symptoms and triggers of CU was collected by a trained member of our research team at study entry. All cohort patients had dermatographism. None of our patients presented only for dermatographism. Dermatographism was tested using a previously described standard diagnostic test where there was moderate stroking of the skin with a wooden spatula [9]. A ques- tionnaire was conducted to query patients on the management of their CIndU, as well as the symptoms experienced by patients in the 15 days preceding their consultation (e.g., itch, wheals, eye swelling, mouth swelling, bothersome symptoms, and medication side effects). The urticaria control test (UCT), a previously validated tool for CIndU [12], was filled for all CIndU patients at study entry and at each follow-up visit. As demonstrated in a recent review, the UCT has high reliability and validity levels [13]. The UCT is not yet formally validated for a pediatric population younger than 12 years. For this age-group, parents/guardians filled UCT forms for their children. In patients who were able to assist, parents/guardians filled it together.

A follow-up questionnaire was conducted with patients over the phone or at consultation, querying on resolution and management of CIndU at a 6-month interval. Descriptive statistics were used to describe the demographics and clinical characteristics.

\section{Assessment of Natural History of the Disease}

CIndU resolution was defined as the absence of hives for at least 1 year without treatment (the date of resolution being the last day of active hives), as described in previous studies [14]. All patients were followed up prospectively and assessed for disease resolution. In case of loss to follow-up, patients were called on an annual basis to verify if the disease was still persistent.

Consenting patients underwent measurements for complete blood counts, baseline levels of C-reactive protein (CRP), total immunoglobulin E (IgE), and tryptase. The patients underwent complementary investigations (e.g., serological tests for antinuclear antibodies and skin biopsy) only in the presence of clinical signs suggestive of an autoimmune disease (e.g., symptoms or arthritis, the presence of nonurticarial rash, or recurrent fever). Basophil activation tests using CD63 marker expression were performed by flow cytometry. Values above $1.8 \%$ of basophil activation were considered elevated, as previously described [15].

\section{Statistical Analysis}

All statistical analyses were performed using $\mathrm{R}$ version 3.2.2. ( $\mathrm{R}$ Core Team [2013]; R: a language and environment for statistical computing; R Foundation for Statistical Computing, Vienna, Austria). Percentages and differences of percentages with $95 \%$ confidence intervals (CIs) were used to summarize patient demographics (age and sex), comorbidities (presence of asthma, atopic dermatitis, hay fever, allergies, anaphylaxis, autoimmune diseases, and autoinflammatory diseases), management (antihistamines, steroids, and omalizumab), and symptoms. Univariate and multivariate logistic regression models were used to determine factors associated with management of CIndU (having a controlled form of CIndU and the need to use of omalizumab to control symptoms, sociodemographic factors, clinical characteristics of urticaria, IgE levels, and tryptase level) and to determine factors associated with resolution of CIndU (age, sex, angioedema, triggers, having a controlled form of CIndU, laboratory findings including basophil count, CRP levels, and comorbidity). The following variables were dichotomized: UCTs with scores $\geq 12$ were defined as well controlled and $<12$ as poorly controlled [16], basophil counts at levels that were detectable or undetectable at $<0.01 \mu \mathrm{g} / \mathrm{L}$, and CD63 levels higher than $1.8 \%$, versus equal or lower, as have been previously described [15]. Age, tryptase levels, and antihistamine dosage were used as continuous variables. 
Fig. 1. Forms of CU. CU, chronic urticaria.

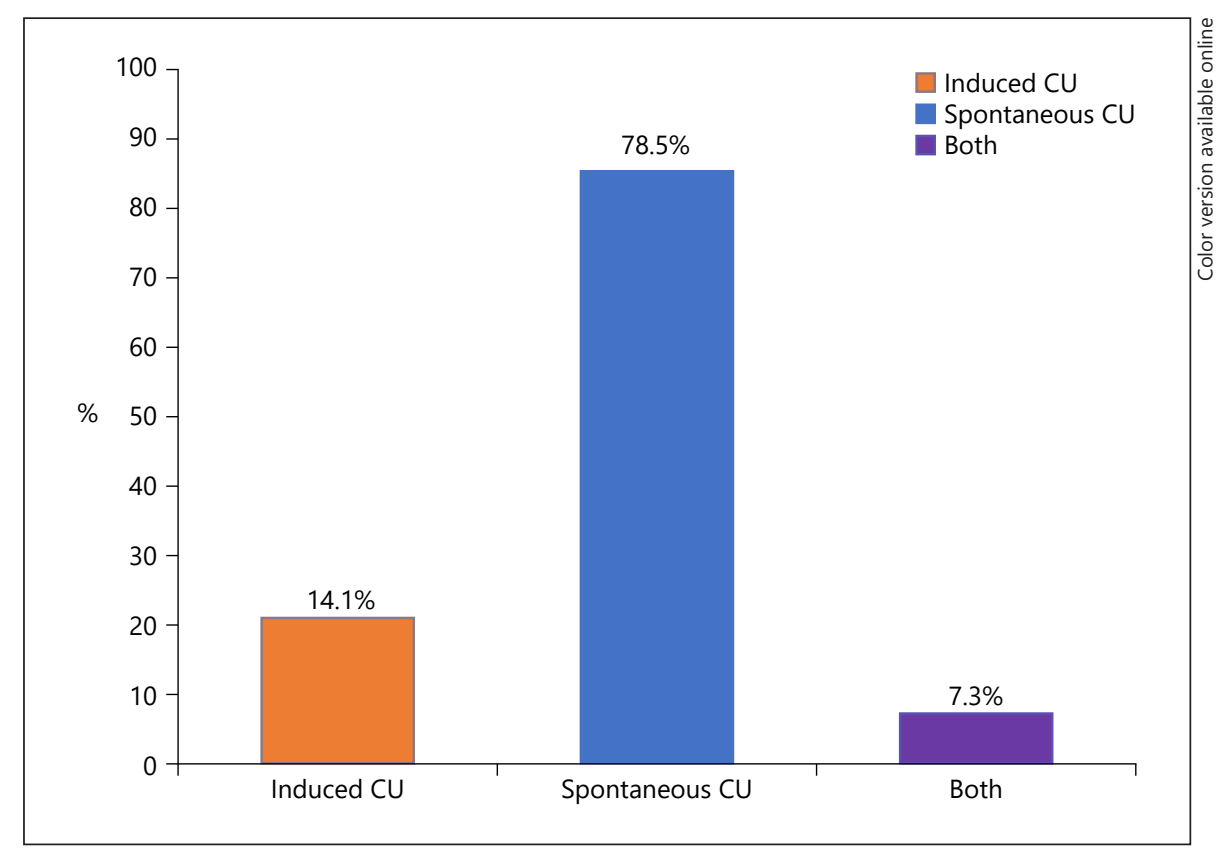

\section{Results}

\section{Demographics (Table 1)}

From June 2013 to July 2019 at the $\mathrm{MCH}, 217$ pediatric patients with CU were recruited, of which $49.3 \%$ were male, with a median age of 9.4 years (interquartile range [IQR] 4.9, 13.6). Among all participants, 29.3\% were diagnosed with CIndU, whereas $84.8 \%$ received a CSU diagnosis; $7.3 \%$ of patients were diagnosed with both CIndU and CSU (Fig. 1). Sixty-four patients presented with CIndU, of which $52 \%$ were male, with a median age of 12.5 years (IQR 7.3, 15.9). Among all CIndU cases, $17 \%$ of patients also presented with angioedema.

Cold CU and cholinergic CU were the most common forms of CIndU (62 and 42\%, respectively). Solar-induced CU affected $6 \%$ of patients, and pressure-induced CU accounted for $3 \%$ of all CIndU cases (some patients had $>1$ form of CIndU). Among all cases of physical CIndU (47 patients) and cold affected $81 \%$, whereas solar-induced accounted for $9 \%$ and pressure-induced represented $4 \%$. Around 10 patients (16\%) with CIndU presented with both cold CU and cholinergic CU.

\section{Comorbidities (Table 2)}

Of all patients with CIndU, one-quarter had an allergic comorbidity. The most common allergic disease was asthma (28\%), followed by atopic dermatitis (19\%) and allergic rhinitis (8\%). Among all cases of CIndU, almost
Table 1. Demographics and forms of CIndU

\begin{tabular}{lc}
\hline Characteristic & $N=64, n(\%)$ \\
\hline Male & $33(52)$ \\
Age, years, median (IQR) & $12.5(7.3,15.9)$ \\
Subtypes of CIndU (some patients had more than one form) \\
$\quad$ Cold-induced & $40(62)$ \\
Cholinergic & $27(42)$ \\
Solar & $10(6)$ \\
Pressure & $2(3)$ \\
Cholinergic and cold-induced & $10(16)$ \\
Family history, $n(\%)$ & \\
$\quad$ Allergic disease & $19(30)$ \\
CU & $6(9)$ \\
\hline
\end{tabular}

Characteristics of CIndU. CIndU, chronic inducible urticaria; $\mathrm{CU}$, chronic urticaria; IQR, interquartile range.

$5 \%$ also had an autoimmune disease, the most common of which was thyroid disease. Nineteen patients (30\%) had family history of allergic disease.

\section{Serologic Markers}

Of the 64 CIndU patients recruited, 38 (59\%) patients consented to baseline blood samples. All of these cases presented with normal tryptase levels. Only $2 \%$ had elevated eosinophil counts. Basophil counts were undetectable in $48 \%$ of CIndU cases. CRP levels were elevated in 
Table 2. Patient clinical characteristics (comorbidities and treatment)

\begin{tabular}{|c|c|c|c|c|c|}
\hline Comorbidity and management & $\begin{array}{l}\text { CIndU } \\
(N=64), n(\%)\end{array}$ & $\begin{array}{l}\text { Cold-induced } \\
(N=40), n(\%)\end{array}$ & $\begin{array}{l}\text { Pressure-induced } \\
(N=2), n(\%)\end{array}$ & $\begin{array}{l}\text { Cholinergic } \\
(N=26), n(\%)\end{array}$ & $\begin{array}{l}\text { Solar-induced } \\
(N=4), n(\%)\end{array}$ \\
\hline Male & $33(52)$ & $20(50)$ & $1(50)$ & $16(62)$ & $1(25)$ \\
\hline \multicolumn{6}{|l|}{ Comorbidities } \\
\hline Asthma & $18(28)$ & $11(28)$ & 0 & $7(27)$ & $1(25)$ \\
\hline Atopic dermatitis & $12(19)$ & $6(15)$ & $1(50)$ & $3(12)$ & $1(25)$ \\
\hline Food allergy & $8(13)$ & $4(10)$ & 0 & $4(15)$ & 0 \\
\hline Autoimmune disease & $3(5)$ & $2(5)$ & 0 & $1(4)$ & $1(25)$ \\
\hline Autoinflammatory disease & $1(2)$ & $1(3)$ & 0 & 0 & 0 \\
\hline Antihistamines & $46(72)$ & $30(75)$ & $1(50)$ & $5(19)$ & $3(75)$ \\
\hline Antihistamines $2 \times$ & $10(16)$ & $7(18)$ & $1(50)$ & $5(19)$ & 0 \\
\hline Antihistamines $4 \times$ & $2(3)$ & $2(5)$ & 0 & 0 & 0 \\
\hline
\end{tabular}

Comorbidities and management according to the subtype of CIndU. CIndU, chronic inducible urticaria.

Table 3. Urticaria control test scores based on the treatment received

\begin{tabular}{llll}
\hline & $\begin{array}{l}\text { Antihistamines } \\
\text { alone } \\
(N=56), n(\%)\end{array}$ & $\begin{array}{l}\text { Steroids with } \\
\text { antihistamines } \\
(N=1), n(\%)\end{array}$ & $\begin{array}{l}\text { Omalizumab with } \\
\text { antihistamines } \\
(N=7), n(\%)\end{array}$ \\
\hline Male, \% & $30(54)$ & $1(100)$ & $3(43)$ \\
Well-controlled UCT score, \% & $44(79)$ & 0 & 0 \\
Antihistamine dosage, mg, median (IQR) & $5.0(1.0,10.0)$ & $5.0(2.5,10.0)$ & $10.0(5.0,17.5)$ \\
\hline
\end{tabular}

Characteristics and UCT scores were based on management. Well-controlled UCT score: scores $\geq 12$. UCT, urticaria control test; IQR, interquartile range.

5 patients (8\%) (UCT scores at the first visit Table 3; see online suppl. Table 1; for all online suppl. material, see www.karger.com/doi/10.1159/000514757).

Mean UCT scores at the first visit was 10.3 (IQR 8.0, 13.0). Well-controlled CIndU at study entry was associated with cold-induced CIndU, while controlling for age, sex, tryptase levels, and elevated CD63 (online suppl. Table 1).

Management (Tables 2, 3; online suppl. Tables 2, 3)

The majority of CIndU cases (72\%) were controlled with a standard dose of second-generation antihistamines (taken a few days before and during exposure to the anticipated triggering factor at standard doses according to age). An additional $16 \%$ of CIndU patients required doubling of antihistamines dosage. More specifically, $18 \%$ of cold-induced CIndU, $50 \%$ of pressure-induced
CIndU, and 19\% of cholinergic CU required twice the standard dose. Only 3\% of CIndU patients required 4 times the standard dose of antihistamines, all of whom suffered only from cold-induced CU. The most commonly used antihistamine for CIndU patients was cetirizine (48\%) (online suppl. Table 2).

Around $85 \%$ of patients were treated with antihistamines alone, whereas $2 \%$ ( 1 patient with cold-induced $\mathrm{CU})$ required short-term systemic corticosteroids for the management of acute exacerbations. Seven patients (11\%) required omalizumab, of which 2 had both cold-induced and cholinergic $\mathrm{CU}$ and 1 patient had pressure-induced CU. All of them were treated concomitantly with secondgeneration antihistamines (Table 2).

The most used antihistamine was cetirizine for CIndU-diagnosed patients (47.62\%). Dosage of antihista- 
mines had a median value of 5.00 (IQR: $1.00,10.00) \mathrm{mg}$ in cases managed by antihistamines alone and 10.00 (IQR: $5.00,17.50) \mathrm{mg}$ in cases managed by omalizumab and antihistamines. Omalizumab doses ranged from 150 to $300 \mathrm{mg}$, with a median value 300 (IQR: 150,300 ) $\mathrm{mg}$. The only patient treated with corticosteroids was prescribed $5.00 \mathrm{mg}$ of prednisone.

More than three-quarters of cases managed by antihistamines alone presented with well-controlled UCT scores. One case managed by corticosteroids for exacerbation had a poor control test score. All cases managed by omalizumab and antihistamines also presented with poor UCT scores at study entry (Table 3 ).

Omalizumab treatment was less likely among patients who were well-controlled according to UCT scores (scores $\geq 12$ ) (adjusted odds ratio [aOR] 0.83, 95\% CI 0.71, 0.98 ) while controlling for age, sex, triggers of CIndU, elevated tryptase levels, detectable basophil count, IgE levels, detectable eosinophilia, and presence of allergic comorbidities (online suppl. Table 3). Additional 5\% were managed by first-generation antihistamines (3 suffered from cold-induced CU, and 1 suffered from solar-induced CU).

\section{Disease Resolution (Table 4; online suppl. Table 4)}

Of the 64 patients included in the study, 24 of them resolved and 11 patients (17.2\%) were lost to follow-up. The crude resolution rate was $45.3 \%$ (95\% CI 33.1-57.5\%), based on the per-protocol population within the 6-year course of the study. Assuming a worst-case scenario with failure for all loss of follow-ups, the resolution rate over a 6-year period declines to $37.5 \%$ (95\% CI 25.6-49.4\%).

Three-quarters of resolved cases had cold-induced CIndU (75\%) compared to $29 \%$ who had cholinergic CU ( 2 of these cases suffered from both cold-induced and cholinergic $\mathrm{CU})$. No resolved cases had pressure-induced CIndU, and $4 \%$ of resolved cases had solar-induced CIndU. In the 24 resolved cases of CIndU, 14 patients had consented to blood samples at baseline. Of these, 6 had elevated CD63 count (43\%) and 7 had increased IgE levels (50\%). All 14 patients had detectable basophil levels, one had elevated CRP levels, and 2 had detectable eosinophil counts (online suppl. Table 4).

Resolution was more likely in patients who presented with well-controlled UCT scores (scores $\geq 12$ ) (aOR, 1.8, 95\% CI 1.4-2.2) and elevated CD63 counts, as well as patients suffering from thyroid comorbidity (aOR 2.1, 95\% CI 2.1-2.2 and aOR 1.4 95\% CI 1.1-1.7, respectively), while controlling for age, sex, presence of angioedema, triggers of CIndU, tryptase levels, IgE levels, and detect-
Table 4. Odds ratios estimated from logistic regression for resolution of CIndU

\begin{tabular}{lll}
\hline Variable & $\begin{array}{l}\text { Univariate } \\
\text { OR }(95 \% \mathrm{CI})\end{array}$ & $\begin{array}{l}\text { Multivariate } \\
\text { OR }(95 \% \mathrm{CI})\end{array}$ \\
\hline Sex & $0.9(0.8,1.3)$ & $0.9(0.8,1.2)$ \\
Age at baseline, years & $1.3(1.1,1.7)$ & $0.9(0.9,1.0)$ \\
Well-controlled UCT baseline & $1.7(1.4,2.1)$ & $1.8(1.4,2.2)$ \\
Presence of angioedema & $1.4(1.0,2.1)$ & $1.9(0.8,4.9)$ \\
Cold-induced & $1.3(0.9,1.6)$ & $0.9(0.8,1.2)$ \\
Cholinergic & $0.8(0.7,1.1)$ & $1.0(0.2,5.5)$ \\
Pressure-induced & $0.7(0.3,1.4)$ & $1.2(0.4,3.8)$ \\
Solar-induced & $0.9(0.5,1.4)$ & $0.5(0.4,0.6)$ \\
Elevated tryptase & $0.9(0.9,1.0)$ & $0.9(0.8,1.1)$ \\
Elevated CRP & $0.9(0.8,1.1)$ & $1.0(0.7,1.7)$ \\
Thyroid comorbidity & $0.7(0.3,1.8)$ & $1.4(1.1,1.7)$ \\
Detectable basophil count & $1.0(0.8,1.4)$ & $1.4(0.4,2.9)$ \\
Elevated CD63 & $1.0(0.9,1.1)$ & $2.1(2.1,2.2)$ \\
Elevated IgE levels & $1.0(0.9,1.0)$ & $1.0(0.9 .1 .0)$ \\
Detectable eosinophilia count & $1.9(0.9,3.8)$ & $1.8(0.8,3.9)$ \\
& &
\end{tabular}

Univariate and multivariate logistic regression models to determine factors associated with resolution of CIndU. Well-controlled UCT: scores $\geq 12$. CIndU, chronic inducible urticaria; CRP, C-reactive protein; UCT, urticaria control test; IgE, immunoglobulin $\mathrm{E}$; CI, confidence interval.

able eosinophilia (Table 4). While controlling for these same factors, resolution was less likely in patients with solar-induced CIndU (aOR 0.5, 95\% CI 0.5, 0.6).

\section{Discussion}

This is the largest North American pediatric study to evaluate current management of CIndU in a pediatric population. Our study reveals that cold and cholinergic forms account for the majority of CIndU cases and that the rate of resolution is relatively low. Further, our data support the use of second-generation antihistamines to control CIndU and the use of omalizumab in more refractory cases.

Current guidelines state that the goal of CIndU management should include the overall control of symptoms as well as disease remission [1]. The first line of treatment for urticaria in a pediatric population includes avoidance of triggers and exacerbating factors when possible and nonsedating second-generation $\mathrm{H} 1$ antihistamine [17]. When first-line treatment does not successfully manage CU symptoms, guidelines recommend that second line of treatment should be updosing of antihistamines up to 4 
times [14]. Clinical trials have supported the efficacy of these antihistamines in CU treatment [18]. The majority of patients in our study were managed with second-generation antihistamines alone. However, $15.6 \%$ of patients required updosing of regular antihistamine dosage, in concord with recent findings demonstrating that patients diagnosed with CIndU are less likely to respond to standard doses of antihistamines than CSU patients [5]. The increased use of second-generation antihistamines is attributed to recent studies concluding that first-generation antihistamines are no longer recommended in allergic treatments, specifically in pediatric populations, due to higher risk of side effects including secondary anticholinergic effects, reduced rapid eye movement sleep, impaired learning, and reduced work efficiency $[19,20]$. Our results are consistent with those of studies suggesting that second-generation antihistamines are effective and safe for the treatment of CIndU in children [14]. In our study, only 4 cases were treated with first-generation antihistamines (due to request from the patients' caregivers).

Finally, guidelines recommend omalizumab as a thirdline treatment for CSU in adults. A consensus-based algorithm established in 2018 placed omalizumab above cyclosporine A for the management of CU [21]. While the use of omalizumab in pediatric CIndU is off-label, many prior reports suggest safety and efficacy in this population [22-24]. In our study, around $10 \%$ of CIndU cases required omalizumab to control their symptoms. Wellcontrolled UCT scores at study entry were less likely in cases who were prescribed omalizumab, reflecting that only more severe forms required omalizumab for management [1]. There was no association between omalizumab treatment and types of triggers of CIndU. However, a systematic review reported that omalizumab has been proven to improve quality of life in CIndU patients but points out that stronger evidence is available for coldinduced CIndU and solar-induced CIndU [22]. This did not seem to have an impact on the cohort studied likely due to the relatively small sample size of the different subgroups of CIndU.

Only 1 patient required the use of corticosteroids during exacerbations of cold-induced CU. The prescription of corticosteroids in the management of $\mathrm{CU}$ is required in cases refractory to antihistamines for a limited period of time $[21,25]$. The UCT score in the case managed by corticosteroids during exacerbations demonstrated that this case had poor control at study entry, thus confirming that corticosteroids have been prescribed for management of more severe cases of $\mathrm{CU}$, as recommended by current guidelines (third-line treatment) [1]. Corticoste- roid side effects are numerous and can be potentially dangerous, such as weight gain, changes in appetite, nausea, and insomnia/fatigue, and therefore should be reserved for severe and refractory cases of $\mathrm{CU}$ and for exacerbations alone, as is the case in our cohort [26]. Furthermore, well-controlled UCT scores were associated with coldinduced CIndU perhaps because this trigger is more controllable (e.g., protective closing and avoidance of immersion into cold bodies of water) [27].

When analyzing the natural history of CIndU, our results are slightly lower than those reported in adult and pediatric studies that have found the rate of resolution in general CU to be in the range of $50 \%$ of cases in 5 years $[28,29]$. The resolution rate over 5 years of a smaller pediatric CIndU cohort reported results that were similar to our own (38.4\%) [5]. Our results are in line with other published findings that CIndU, more specifically physical urticaria, usually has a worse resolution rate than other forms of CU [30]. Well-controlled study entry UCT scores were associated with disease resolution, likely demonstrating that disease severity and control levels affect the natural history of CIndU. Finally, in our cohort, pediatric patients who also had a concomitant thyroid disease were more likely to have resolution of CIndU. Previous case reports have linked resolution of CU with thyroid hormone therapy [31]. Additionally, studies have demonstrated resolution of $\mathrm{CU}$ in patients treated for thyroid disease [32]. Further studies are needed to confirm this association due to the few patients in our cohort with thyroid comorbidity.

The presence of angioedema was reported as $20 \%$ in cold-induced CU. In a study involving adult and pediatric patients with cold-induced urticaria, angioedema was present in $12 \%$ of patients. Symptoms such as angioedema may occur only after exposure to cold, during the rewarming phase [33]. Since there are little data on the mechanism of angioedema in CIndU in pediatric cohorts, it is possible that angioedema presents more frequently in pediatric cases of cold-induced urticaria than adult cohorts [27].

The limitations of this study include a relatively small subgroup for certain triggers, rendering associations with subtypes of CU inconclusive. Further studies are needed to demonstrate the natural history based on subtypes of CIndU. Other limitations include the fact that we could not assess specifically dermatographism as all our patients had some degree of dermographism. In addition, larger studies are required to assess the efficacy and safety of omalizumab in pediatric patients with different types of CIndU. Furthermore, although not vali- 
dated for children, we preferred to use the UCT scores on both adolescents who are able to understand the questions as well as on children where we relied on parent information.

In conclusion, most cases of CIndU were effectively managed by second-generation antihistamines. The use of corticosteroids and omalizumab was restricted to more severe cases of CU. Almost one-fifth of CIndU cases required updosing of antihistamines, confirming previous research findings on lower response rates of CIndU to standard antihistamine doses. The vast majority of antihistamines prescribed in our cohort were second-generation antihistamines, following current guidelines on different level lines of treatment and the step-up approach. The natural history of CIndU resolution in pediatric patients was relatively low but similar to the rate of resolution in adult cohorts and was associated with elevated CD63 levels, as well as thyroid comorbidity and well-controlled UCT scores at study entry.

\section{Statement of Ethics}

The urticaria registry is a cohort study recruiting children with CU. Approval was received by the McGill University Health Centre Research Ethics Board, from the Centre for Applied Ethics
(REB 12-255 GEN, June 3, 2013). Written informed consent was obtained from parents or legal guardians of patients, as well as patients older than 7 years.

\section{Conflict of Interest Statement}

The authors disclose no conflict of interest.

\section{Funding Sources}

No funding was provided for this study.

\section{Author Contributions}

L.M. contributed to the methodology investigation, data analysis, writing of the initial manuscript, and editing of the manuscript. M.B.S. and S.G. were involved in the supervision and oversight, contributed to methodology and data analysis, and reviewing and editing the manuscript. M.L., E.N., S.B., and S.G. contributed to data curation.

\section{Availability of Data and Material}

The data that support the findings of this study are available from the corresponding author upon reasonable request.

\section{References}

1 Sussman G, Hébert J, Gulliver W, Lynde C, Waserman S, Kanani A, et al. Insights and advances in chronic urticaria: a Canadian perspective. Allergy Asthma Clin Immunol. 2015;11(1):7.

2 Del Pozzo-Magaña BR. Chronic urticaria in children: a review. EMJ Dermatol. 2017;5(1): 74-82.

3 Fricke J, Ávila G, Keller T, Weller K, Lau S, Maurer M, et al. Prevalence of chronic urticaria in children and adults across the globe: systematic review with meta-analysis. Allergy. 2020;75(2):423-32.

4 Jo YH, Yoo HW, Kim SH, Kim YM, Kim H-Y. Clinical characteristics and treatment response of chronic spontaneous urticaria according to age: a single-center Korean study. Asian Pac J Allergy Immunol. 2019.

5 Khakoo G, Sofianou-Katsoulis A, Perkin MR, Lack G. Clinical features and natural history of physical urticaria in children. Pediatr Allergy Immunol. 2008;19(4):363-6.

6 Caffarelli C, Paravati F, El Hachem M, Duse M, Bergamini M, Simeone G, et al. Management of chronic urticaria in children: a clinical guideline. Ital J Pediatr. 2019;45(1):101.

7 Dice JP, Gonzalez-Reyes E, Elmets CA. In: Saini SEC, editor. Physical (inducible) forms of urticaria. 2018.
8 Netchiporouk E, Sasseville D, Moreau L, Habel Y, Rahme E, Ben-Shoshan M. Evaluating comorbidities, natural history, and predictors of early resolution in a cohort of children with chronic urticaria. JAMA Dermatol. 2017; 153(12):1236-42.

9 Magerl M, Altrichter S, Borzova E, GiménezArnau A, Grattan CE, Lawlor F, et al. The definition, diagnostic testing, and management of chronic inducible urticarias: the EAACI/ GA(2) LEN/EDF/UNEV consensus recommendations 2016 update and revision. Allergy. 2016;71(6):780-802.

10 Maurer M, Metz M, Bindslev-Jensen C, Bousquet J, Canonica GW, Church MK, et al. Definition, aims, and implementation of GA(2) LEN urticaria centers of reference and excellence. Allergy. 2016;71(8):1210-8.

11 Trevisonno J, Balram B, Netchiporouk E, Ben-Shoshan M. Physical urticaria: review on classification, triggers and management with special focus on prevalence including a metaanalysis. Postgrad Med. 2015;127(6):565-70.

12 Weller K, Groffik A, Church MK, Hawro T, Krause K, Metz M, et al. Development and validation of the urticaria control test: a patient-reported outcome instrument for assessing urticaria control. J Allergy Clin Immunol. 2014;133(5):1365-6.
13 Maurer M, Eyerich K, Eyerich S, Ferrer M, Gutermuth J, Hartmann K, et al. Urticaria: collegium internationale allergologicum (CIA) update 2020. Int Arch Allergy Immunol. 2020;181(5):321-33.

14 Ben-Shoshan M, Grattan CE. Management of pediatric urticaria with review of the literature on chronic spontaneous urticaria in children. J Allergy Clin Immunol Pract. 2018 JulAug;6(4):1152-61.

15 Netchiporouk E, Moreau L, Rahme E, Maurer M, Lejtenyi D, Ben-Shoshan M. Positive CD63 basophil activation tests are common in children with chronic spontaneous urticaria and linked to high disease activity. Int Arch Allergy Immunol. 2016;171(2):81-8.

16 Kocatürk E, Can PK, Akbas PE, Copur M, Degirmentepe EN, Kiziltac K, et al. Management of chronic inducible urticaria according to the guidelines: a prospective controlled study. J Dermatol Sci. 2017;87(1):60-9.

17 Simons FE; Group EPoAiACS. H1-antihistamine treatment in young atopic children: effect on urticaria. Ann Allergy Asthma Immunol. 2007;99(3):261-6.

18 Golightly LK, Greos LS. Second-generation antihistamines: actions and efficacy in the management of allergic disorders. Drugs. 2005;65(3):341-84. 
19 Church M, Maurer M, Simons F, BindslevJensen C, van Cauwenberge P, Bousquet J, et al. Risk of first-generation H1-antihistamines: a GA2LEN position paper. Allergy. 2010;65(4):459-66.

20 Tannenbaum C, Paquette A, Hilmer S, Holroyd-Leduc J, Carnahan R. A systematic review of amnestic and non-amnestic mild cognitive impairment induced by anticholinergic, antihistamine, GABAergic and opioid drugs. Drugs Aging. 2012;29(8):639-58.

21 Zuberbier T, Aberer W, Asero R, Abdul Latiff $\mathrm{AH}$, Baker D, Ballmer-Weber B, et al. The EAACI/GA ${ }^{2}$ LEN/EDF/WAO guideline for the definition, classification, diagnosis and management of urticaria. Allergy. 2018;73(7): 1393-414.

22 Maurer M, Metz M, Brehler R, Hillen U, Jakob T, Mahler V, et al. Omalizumab treatment in patients with chronic inducible urticaria: a systematic review of published evidence. J Allergy Clin Immunol. 2018;141(2): 638-49.
23 Ivyanskiy I, Sand C, Thomsen SF. Omalizumab for chronic urticaria: a case series and overview of the literature. Case Rep Dermatol. 2012;4(1):19-26.

24 Brodská P, Schmid-Grendelmeier P. Treatment of severe cold contact urticaria with omalizumab: case reports. Case Rep Dermatol. 2012;4(3):275-80.

25 Vonakis BM, Saini SS. New concepts in chronic urticaria. Curr Opin Immunol. 2008; 20(6):709-16.

26 Buchman AL. Side effects of corticosteroid therapy. J Clin Gastroenterol. 2001;33(4): 289-94.

27 Stepaniuk P, Vostretsova K, Kanani A. Review of cold-induced urticaria characteristics, diagnosis and management in a Western $\mathrm{Ca}$ nadian allergy practice. Allergy Asthma Clin Immunol. 2018;14(1):1-7.
28 Sahiner UM, Civelek E, Tuncer A, Yavuz ST, Karabulut E, Sackesen C, et al. Chronic urticaria: etiology and natural course in children. Int Arch Allergy Immunol. 2011;156(2):22430.

29 Taylor-Black SA, Wang J. Chronic urticaria: etiology and natural course in children. Pediatrics. 2012;130(Suppl 1):S22-3.

30 Engstrom J, Neher JO, St Anna L. What is the prognosis for patients with chronic urticaria? Clin Inquiries. 2011.

31 Bangash SA, Bahna SL. Resolution of chronic urticaria and angioedema with thyroxine. $\mathrm{Pa}$ per presented at: Allergy \& Asthma Proceedings. 2005.

32 Gaig P, García-Ortega P, Enrique E, Richart C. Successful treatment of chronic idiopathic urticaria associated with thyroid autoimmunity. J Investig Allergol Clin Immunol. 2000; 10(6):342.

33 Maltseva N, Borzova E, Fomina D, Bizjak M, Terhorst-Molawi D, Košnik M, et al. Cold urticaria what we know and what we do not know. Allergy. 2020. 